\title{
Genome-wide identification for genes involved in sodium dodecyl sulfate toxicity in Saccharomyces cerevisiae
}

\author{
Chunlei $\mathrm{Cao}^{1,2}$, Zhengfeng $\mathrm{CaO}^{3}$, Peibin $\mathrm{Yu}^{1,2}$ and Yunying Zhao ${ }^{1,2^{*}}$
}

\begin{abstract}
Background: Sodium dodecyl sulfate (SDS) is one of the most widely used anionic alkyl sulfate surfactants. Toxicological information on SDS is accumulating, however, mechanisms of SDS toxicity regulation remain poorly understood. In this study, the relationship between the SDS-sensitive mutants and their intracellular ROS levels has been investigated.

Results: Through a genome-scale screen, we have identified 108 yeast single-gene deletion mutants that are sensitive to $0.03 \% \mathrm{SDS}$. These genes were predominantly related to the cellular processes of metabolism, cell cycle and DNA processing, cellular transport, transport facilities and transport routes, transcription and the protein with binding function or cofactor requirement (structural or catalytic). Measurement of the intracellular ROS (reactive oxygen species) levels of these SDS-sensitive mutants showed that about 79\% of SDS-sensitive mutants accumulated significantly higher intracellular ROS levels than the wild-type cells under SDS stress. Moreover, SDS could generate oxidative damage and up-regulate several antioxidant defenses genes, and some of the SDSsensitive genes were involved in this process.
\end{abstract}

Conclusion: This study provides insight on yeast genes involved in SDS tolerance and the elevated intracellular ROS caused by SDS stress, which is a potential way to understand the detoxification mechanisms of SDS by yeast cells.

Keywords: Saccharomyces cerevisiae, SDS, Genetic screening, Genomics, ROS

\section{Background}

Surfactants are organic pollutants distributed widely in the current environment, and their toxicity has caused widespread concern. One of the synthetic anionic surfactants, sodium dodecyl sulfate (SDS), or sodium lauryl sulfate (SLS), a product that consists of approximately $70 \%$ sodium dodecyl sulfate and $30 \%$ sodium tetradecyl sulfate, with the formula of $\mathrm{CH} 3(\mathrm{CH} 2)_{11} \mathrm{OSO}_{3} \mathrm{Na}$, has been used in many cleaning and hygiene products such as liquid soaps, shampoos, bubble baths, shower gels, and nearly all toothpastes. SDS is also used in

\footnotetext{
* Correspondence: yunyingzhao@jiangnan.edu.cn

${ }^{1}$ National Engineering Laboratory for Cereal Fermentation Technology (NELCF), Jiangnan University, 1800 Lihu Road, Wuxi 214122, Jiangsu, China

${ }^{2}$ Jiangsu Provincial Research Center for Bioactive Product Processing

Technology, Jiangnan University, 1800 Lihu Road, Wuxi 214122, Jiangsu,

China

Full list of author information is available at the end of the article
}

pharmaceutical and food products, as well as in industiral and laboratory applications, i.e., SDS can form complexes with protein through hydrophobic interactions and thus be used in polyacrylamide gel electrophoresis to determine the molecular weight of proteins $[1,2]$. The concentration of SDS found in consumer products varies by product and manufacturer but typically ranges from 0.01 to $50 \%$ in cosmetic products and 1 to $30 \%$ in cleaning products [3]. The lethal dose, 50\% (LD50) for SDS is $0.8-1.10 \mathrm{~g} / \mathrm{kg}$ in rats, SDS concentrations $>2 \%$ are considered irritating to normal skin in human patch testing, and $>5 \%$ causes depression, labored breathing, diarrhea, and death (four out of 20 animals) [2].

Safety concerns with SDS application in human include carcinogenicity, skin and eye irritation, and aphthous ulcers. The toxicity of SDS has been demonstrated in bacteria, microalgae, crustaceans, echinoderms, rats, humans and carp. The basis of SDS toxicity seems to be 
mainly related to the alteration of the cellular ionic balance caused by cellular membrane permeability alterations and to the induction of oxidative stress, that can generate other physiological and biochemical stresses [4]. SDS elicits both physical and biochemical effects on cells, with the membrane the primary target structure, and considered as a typical cell wall perturbing agent. Effects are concentration dependent and range from loss of barrier function and increased permeability to complete cell lysis. It is suggested that SDS causes elevated the glutathione production, lipid peroxidation as well as changes in carbon metabolism [5], leading to altered cell membrane stability and permeability as well as indirectly to increased accessibility of cell wall [6]. Yeast cell wall serves crucial functions in protecting against osmotic shock stress and mechanical steess, maintaining cell shape, as well as serving a sacffold for cell-surface proteins [7]. SDS interrupts cell membranes and then triggers the Cell Wall Integrity (CWI) signaling pathway, a kinase cascade to maintain cell integrity and can be activated by chemicals that damage the cell wall and membrane in buding yeast [8]. For example, the Slt2/Mpk1, a mitogen-activated protein (MAP) kinase, can be phosphorylated and thus activated by impaired cell integrity [9]. However, deatiled mechanisms of SDS toxicity in microorganisms or higher eukaryotes are poorly understood.

Yeast has been previously used to demonstrate the effect of SDS on biological membranes, showing that micelles of SDS may penetrate the membrane through pores in the yeast cell wall and destroy the membrane [10]. In defense against SDS surplus, yeast cells increase the expression levels of genes involved in oxidative stress which might be caused by its effect on membrane structure, carbon metabolism, or DNA repair [2]. Reactive oxygen spesies (ROS) play an important role in inducing cell death or apotosis in yeast cells by causing damages to proteins, lipids and DNA [11, 12]. In addtion, ROS could induce cell wall damage in yeast cells lacking mitochondrial DNA, making cells to become more sensitive to of SDS stress [13].

As the simplest eukaryotic organism, the budding yeast Saccharomyces cerevisiae (S. cerevisiae) has been used to identify the mechanism and regulation of metal ion transport [14]. Here, we used S. cerevisiae to explore the SDS effect on eukaryotic cell growth and compared the oxidative stress (reactive oxygen species, ROS) in cultured cells. We have firstly screened the SDSsensitive mutants from the yeast nonessential gene deletion library and identified 108 SDS-sensitive mutants. To evaluate whether SDS generates serious oxidative stress to the SDS-sensitive mutant cells, we have then measured the cellular response of cultured yeast cells to SDS in terms of ROS levels. Specifically, we show that
SDS can induce oxidative stress and that yeast cells eliminate these oxidative damage by elevating the expression levels of the genes coding for antioxidant defenses.

\section{Results \\ An overview of genes involved in the SDS sensitivity of yeast cells}

To investigate the cellular functions required for cell growth under a surplus of SDS, a yeast library of diploid nonessential gene deletion was screened to identify genes involved in the sensitivity to SDS. The results show that 108 gene deletion mutants $(2.3 \%$ of the screened 4757 gene deletion mutants) were identified as sensitive to $0.03 \%$ SDS (Fig. 1 and Table 1). The genotypes of these 108 mutants were confirmed by PCR with the forward primer derived from the promoter region of each correspondent gene and a reverse primer KanMX4$\mathrm{R}$ (Additional file 1: Table S1 and Additional file 2: Fig. S1) derived from the ORF region of KanMX4. The functional categories of these 108 genes are involved in metabolism (17), cell cycle and DNA processing (15), transcription (14), cellular transport, transport facilities and transport routes (28), biogenesis of cellular components (6), cellular communication / signal transduction mechanism (2), protein with binding function or cofactor requirement (structural or catalytic) (10), as well as unclassified proteins (16) (Table 1). Gene Ontology (GO) enrichment analysis result showed that these 108 SDS-sensitive genes were mainly enriched in vacuolar transport, ATP export, and endosomal transport among the top 16 GO terms in cluster groups (Additional file 3: Fig. S2).

\section{Exposure to SDS stress results in ROS generation}

Since SDS had been confirmed to induce the oxidative stress response [2], we next measured the intracellular ROS levels of the 108 SDS-sensitive mutants under $0.015 \%$ SDS treatment. In the wild-type BY4743 cells, the intracellular ROS level was significantly increased under SDS stress (Fig. 2 and Additional file 4: Fig. S3). Interestingly, only six mutants for ARG82, TRP5, GRR1, MSH1, LAS21, and YNL296W of these 108 SDS-sensitive mutants, accumulated lower intracellular ROS levels when treated with $0.015 \%$ SDS than without SDS (The relative ROS levels in these mutants was smaller than 1 ; Fig. 2 and Additional file 4: Fig. S3). It suggested that the above six genes might not be directly involved in the regulation of intracellular ROS levels under SDS stress. Of these 108 SDS-sensitive mutants, 85 mutants accumulated significantly higher intracellular ROS levels under SDS stress compared with wild-type cells (Additional file 4: Fig. S3B and D), indicating that these 85 mutants might respond to lower concentration of SDS and thereby accumulated higher ROS levels than wild 


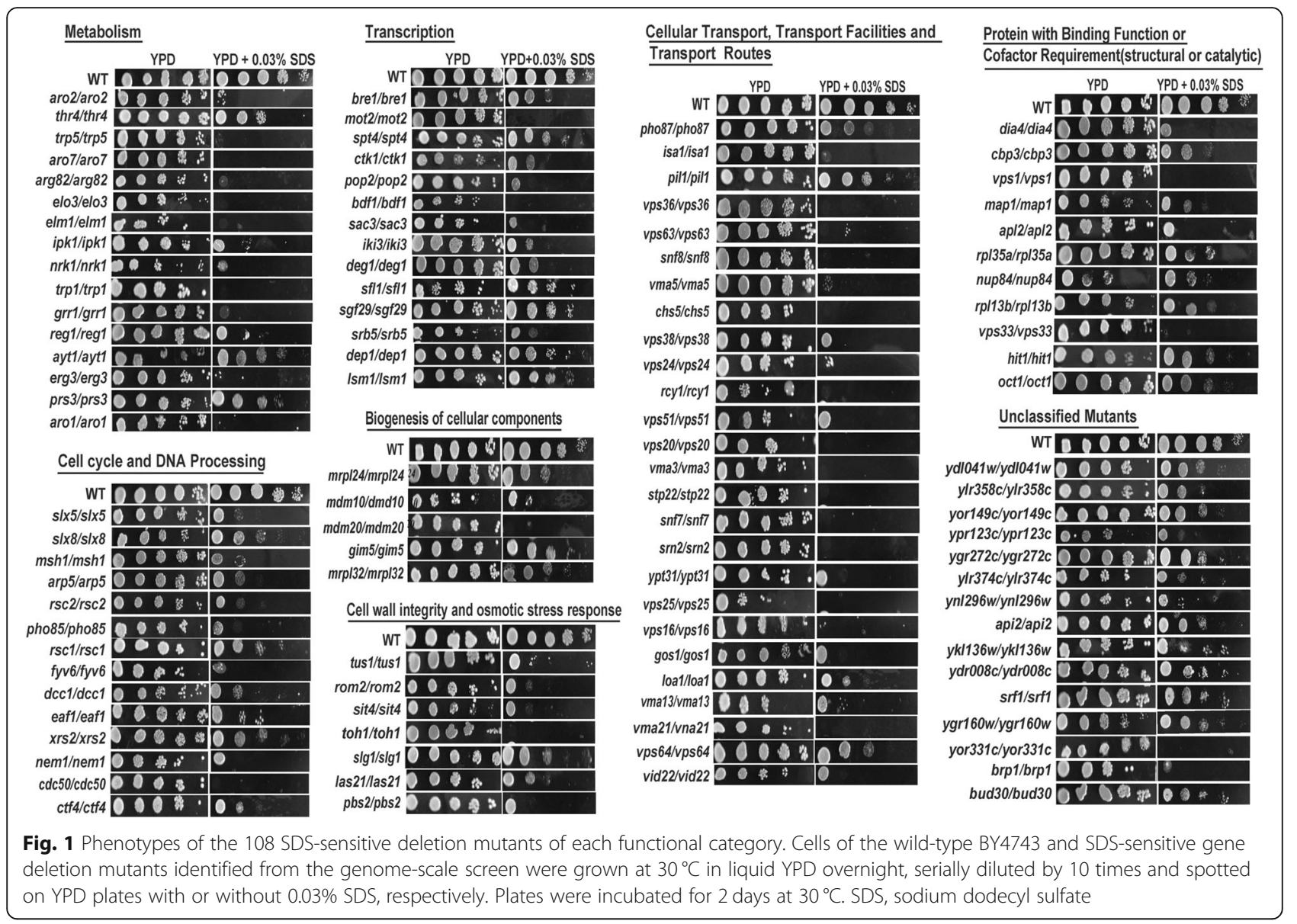

type cells. The rest 23 mutants accumulated similar or lower intracellular ROS levels when treated with SDS compared with wild type cells, although the relative ROS levels in some of these mutants were also very high (Fig.2 and Additional file 4: Fig. S3B and D). Here we showed that mutants for genes related to the functions of metabolism and cellular transport, transport facilities and transport routes were most sensitive to SDS stress (Table 1). We listed some genes as the representative genes of their categories as below.

Genes involved in cellular transport and transport routes are associated with SDS tolerance

The largest functional category of these 108 identified SDS-sensitive genes is the cellular transport, transport facilities and transport routes (Table 1), including 28 genes identified. There are 63 nonessential vacuolar protein sorting (VPS) genes in the genome of $S$. cerevisiae [15]. Notably, 15 mutants for VPS1, VPS16, VPS20, VPS24, VPS22, VPS23, VPS25, VPS32, VPS33, VPS36, VPS37,VPS38, VPS51, VPS63, and VPS64 were identified being sensitive to $0.03 \%$ SDS in the present study (Table 1; Fig. 1). The intracellular ROS levels of these $15 \mathrm{mu}$ tants were all induced by SDS stress, especially in mutants for VPS20, VPS36, VPS63 and VPS25 (Fig. 2). The results suggest that the VPS pathway involved in protein trafficking and membrane fusion plays an important role in the response of yeast cells to SDS stress.

The $\mathrm{H}^{+}$-ATPase localized in the membrane of vacuole (V-ATPase) is composed of the catalytic V1 subcomplex and the proton-translocating membrane V0 subcomplex, playing crucial roles in the organelles acidification and other intracellular activities [16, 17]. In this study, four mutants for VMA3, VMA5, $V M A 13$, and $V M A 21$ were sensitive to $0.03 \%$ SDS (Table 1; Fig. 1). VMA5 and VMA13 encodes the V1 complex subunit $\mathrm{C}$ and $\mathrm{H}[18,19]$, respectively. $V M A 3$ encodes the subunit c of the V0 complex [20]. $V M A 21$ is not an actual component of the V-ATPase complex, but encodes proteins functioned in the assembly of the V-ATPase [21]. These results indicate that the V-ATPase is critical for $S$. cerevisiae cells in responding to SDS in the environment. 
Table 1 Functional categories of 108 genes whose deletion mutants are sensitive to $0.03 \%$ SDS

\begin{tabular}{|c|c|c|c|c|c|c|c|c|}
\hline \multirow{3}{*}{$\frac{\text { Function }}{\text { Metabolism (16) }}$} & \multicolumn{8}{|l|}{ Genes } \\
\hline & PRS3 ${ }^{*}$ & THR4 $4^{* *}$ & $A R G 82^{* * * *}$ & $R E G 1^{* * *}$ & $\mid P K 1^{* * *}$ & $A R O 1^{* * * * * *}$ & $T R P 1^{* * * * *}$ & $\operatorname{TRP5}^{* * * * *}$ \\
\hline & $E L M 1^{* * * * *}$ & AYTI $^{*}$ & $E L O 3^{* * * * *}$ & $A R O 7^{* * * * *}$ & $A R O 2^{* * * *}$ & $N R K 1^{* * * *}$ & $G R R 1^{* * * *}$ & $E R G 3^{* * * *}$ \\
\hline \multirow[t]{2}{*}{ Cell cycle and DNA processing (14) } & $N E M 1^{* * * *}$ & $\operatorname{CDC} 50^{* * * * * *}$ & $S L X 5^{* * *}$ & PHO85 $5^{* * *}$ & $M S H 1^{* * *}$ & $\mathrm{DCCl}^{* * *}$ & $E A F 1^{* *}$ & XRS2 \\
\hline & $\operatorname{RSC} 2^{* * *}$ & $F Y V \sigma^{* * * *}$ & $A R P 5^{* *}$ & $C T F 4^{* *}$ & $\mathrm{RSCl}^{*}$ & $S L X 8^{*}$ & & \\
\hline \multirow[t]{2}{*}{ Transcription (14) } & $B R E I^{* *}$ & $B D F 7^{* * * * *}$ & $D E P 1^{*}$ & $S A C 3^{* * * *}$ & MOT2 $2^{* * * * *}$ & $D E G 1^{* * *}$ & $S F L 1^{*}$ & $P O P 2^{* * * *}$ \\
\hline & $C T K 1^{* * *}$ & $\angle S M 1^{*}$ & $S R B 5^{* * *}$ & SPT4 ${ }^{*}$ & $|K| 3^{* * *}$ & SGF29* & & \\
\hline \multirow{4}{*}{$\begin{array}{l}\text { Cellular transport, transport facilities and transport } \\
\text { routes }(28)\end{array}$} & GOSi $1^{* * * *}$ & PHO87 & VPS $23^{* * * * *}$ & $V M A 3^{* * * * *}$ & YPT31 $1^{* * * *}$ & $L O A 1^{* * *}$ & VMA13 & VPS $22^{* * * * *}$ \\
\hline & VPS36 $6^{* * * * *}$ & VPS38 $8^{* * * *}$ & VPS37 $7^{* * * * *}$ & VPS63 $3^{* * * * *}$ & VPS $24^{* * * *}$ & VPS25 $5^{* * * * *}$ & $R C Y 1^{* * * * *}$ & VPS51 $1^{* * * *}$ \\
\hline & CHS5 ${ }^{* * * * *}$ & VPS32 $2^{* * * * *}$ & $|S A|^{* * * *}$ & PIL $1^{* *}$ & $V M A 5^{* * * *}$ & VPS20 $0^{* * * * *}$ & VPS16 $6^{* * * * *}$ & $V P S 1^{* * * * *}$ \\
\hline & VPS33 $3^{* * * * *}$ & VPS64 & $V M A 21^{* * * * *}$ & VID $22^{* * * *}$ & & & & \\
\hline \multirow{2}{*}{$\begin{array}{l}\text { Protein with binding function or cofactor } \\
\text { requirement (structural or catalytic) (9) }\end{array}$} & $D I A 4^{* * * *}$ & $R P L 35 A^{* *}$ & NUP84 & $A P L 2^{* * * *}$ & MAP $1^{* *}$ & $\operatorname{RPL13B^{**}}$ & $\mathrm{CBP}^{* *}$ & $\mathrm{HITT}^{*}$ \\
\hline & $\mathrm{OCT}{ }^{* *}$ & & & & & & & \\
\hline Biogenesis of cellular components (5) & $M D M 10^{* * *}$ & MRPL32* & MRPL24 $4^{* *}$ & GIM5* & $M D M 20^{* * * * *}$ & & & \\
\hline Cell wall integrity and osmotic stress response (7) & TUSi $1^{* * *}$ & $R O M 2^{* * *}$ & SIT4 $4^{* * *}$ & $\mathrm{TOH}^{* * * * *}$ & $S L G I^{*}$ & $\operatorname{LAS} 21^{* *}$ & $P B S 2^{* * * *}$ & \\
\hline \multirow[t]{2}{*}{ Unclassified proteins (15) } & $S R F 7^{*}$ & YDL041W* & $Y L R 358 C^{* *}$ & YNL296W ${ }^{* * *}$ & YPR123C & YDR149C & YOR331C $C^{* * * * *}$ & YGR272C \\
\hline & YLR374C ${ }^{*}$ & YKL136W* & YGR160W* & $B R P 1^{* * * *}$ & $B \cup D 30^{*}$ & $A P I 2^{* *}$ & YDR008C $C^{*}$ & \\
\hline
\end{tabular}

The number of asterisks represents SDS-sensitivity of different mutants. Mutant with five asterisks was most sensitive to SDS stress, while mutant with one asterisk was least sensitive to SDS

\section{Mutants for genes involved in cell cycle and DNA processing render yeast cells sensitive to SDS stress}

There are 14 genes identified in our study that are involved in cell cycle and DNA processing (Table 1; Fig. 1). The intracellular ROS levels in 13 mutants except the mutants for MSH1 were all increased under SDS stress, especially in mutants for MEM1, PHO85, EAF1 and XRS2 (Fig. 2). SLX5 and SLX8 encode the subunit of Slx5-Slx8 ubiquitin-like modifier (SUMO)-targeted ubiquitin ligase (STUbL) complex [22-24]. Mutants for SLX5 or SLX8 were sensitive to $0.03 \%$ SDS (Table 1 and Fig. 1), suggesting that STUbL complex is involved in SDS tolerance of yeast cells. The small SUMO-targeted ubiquitin ligase complex is a nuclear ubiquitin ligase complex that specifically targets sumoylated proteins. It is formed of homodimers or heterodimers of RING finger protein 4 family ubiquitin ligases and is conserved in eukaryotes [23]. Three genes, MSH1, FYV6 and XRS2, encode three proteins required for the DNA repair process [25-27], has been identified in this study. The other six genes, EAF1, ARP5, RSC1, RSC2, DCC1 and $C T F 4$ associated with chromatin modification, remodeling and cohesion [28-32], are all required for SDS tolerance. The PHO85 gene, coding for a cyclin-dependent kinase Pho85, was screened in our study. The kinase Pho85 is involved in regulating the cellular responses of cell cycle progression, autophagy, response to DNA damage, phosphate and glycogen metabolism, establishment of cell polarity, as well calcium-mediated signaling. Therefore, deletion of the PHO85 cause a decreased resistance to oxidative stress, chemicals, toxin, utilization of carbon and nitrogen [33-37]. In addition, we have identified two genes, NEM1 and CDC50, which are required for normal nuclear envelope morphology and sporulation, or cell division, respectively [38, 39]. Taken together, these results suggests that SDS can affect the cell cycle and DNA processing of $S$. cerevisiae cells.

\section{Genes involved in aromatic amino acid biosynthesis and SDS tolerance}

We have identified mutants for five genes involved in the synthesis of aromatic amino acids, ARO1, ARO2, ARO7, TRP1 and TRP5 that were sensitive to $0.03 \%$ SDS (Table 1; Fig. 1). The intracellular ROS levels in mutants for ARO1, ARO7, TRP1 and TRP5 were all higher than that of wide type cells when the cells were treated with SDS (Additional file 4: Fig. S3B and D). Previously, Aro1 catalyzes steps 2 through 6 in the biosynthesis of chorismate, which is a precursor to aromatic amino acids [23]; Aro2 catalyzes the conversion of 5-enolpyruvylshikimate 3-phosphate (EPSP) to form chorismate; and Aro7 catalyzes the conversion of chorismate to prephenate to initiate the tyrosine/phenylalanine-specific branch of aromatic amino acid biosynthesis [40-42]. Trp1 and Trp5 involved in the synthesis of tryptophan, where Trp1 catalyzes the third step in tryptophan biosynthesis and Trp5 catalyzes the last step of tryptophan biosynthesis $[43,44]$. It was reported previously that trp1-1 cells had a disadvantage in the response to SDS compared to auxotrophy for adenine, histidine, leucine or 


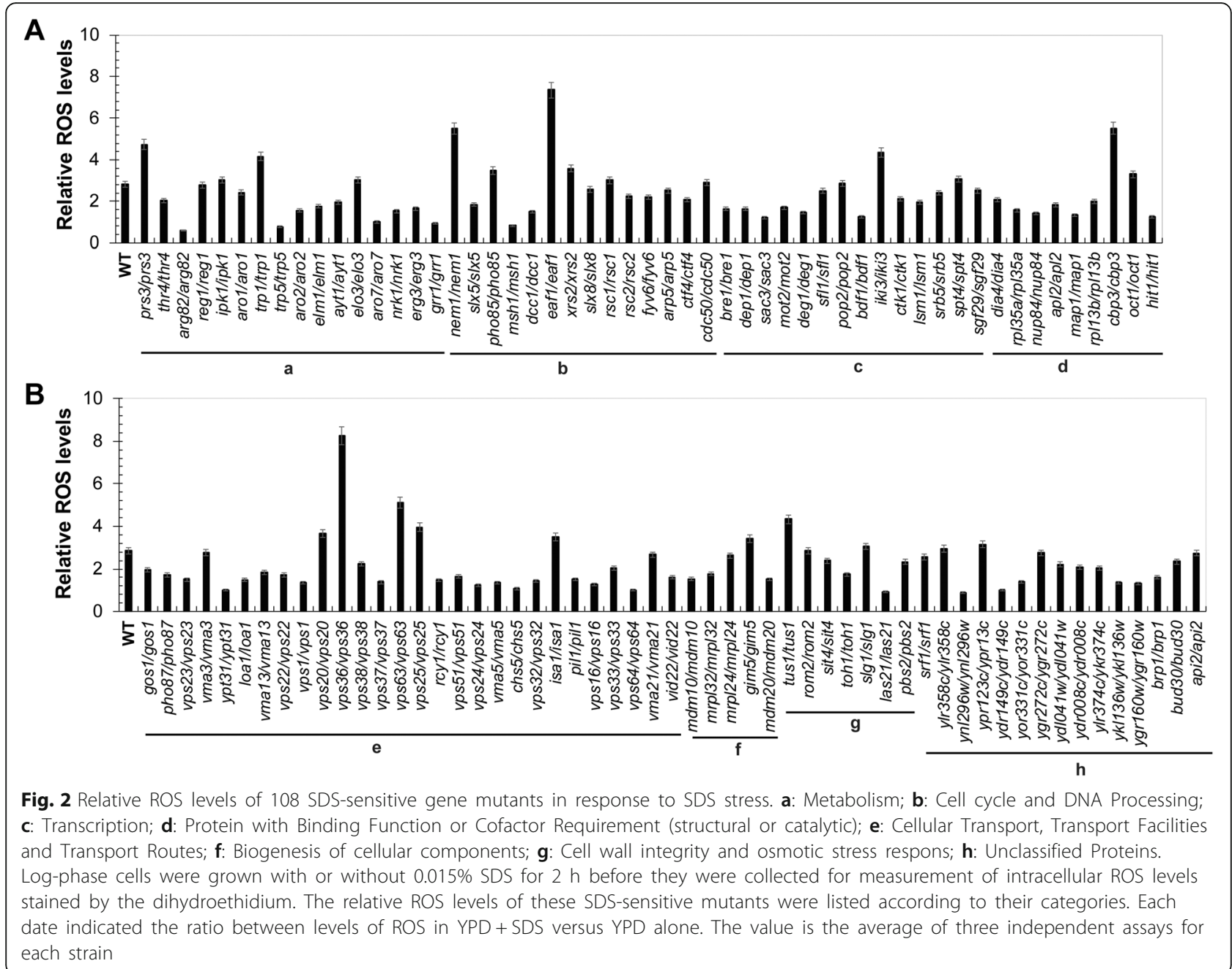

uracil when cells were grown on rich media [45]. They also showed that the cell membrane damage triggered by SDS was independent of CWI (cell wall integrity) signaling and was not a cause of tryptophan starvation. Our present results confirmed this previous findings that tryptophan exhibited protection from membrane disruptions and thus conferred resistance to SDS stress.

\section{SDS generates oxidative stress by regulating the expression of genes involved in redox homeostasis}

The relative ROS levels in 11 mutants for PRS3, TRP1, NEM1, EAF1, IKI3, CBP3, VPS20, VPS36, VPS63, VPS25, and TUS1 were all higher than that of wild-type cells (Fig. 2), indicating that these 11 genes were all important for dealing with the oxidative damage generated by SDS stress. To further confirm these results, we constructed the 11 plasmids expressing the above 11 genes in pRS316 plasmid, respectively, and then transformed them into the corresponding mutants. The growth defect of SDStreatment mutant cells could be suppressed by introducing the expression plasmid back into the corresponding mutants (Fig. 3a), and their intracellular ROS levels were also recovered to that of the wildtype cells (Fig. 3b). Taken together, these results indicate that yeast cells lacking any of the above 11 genes are sensitive to SDS stress, leading to increased intracellular ROS levels.

It was reported that many of the oxidative stress scavenging genes could be induced by SDS stress in a DNA microarray analysis [2]. To investigate whether the deletion of genes PRS3, TRP1, NEM1, EAF1, IKI3, CBP3, VPS20, VPS36, VPS63, VPS25, and TUS1 influence the expression of genes coding for the antioxidant defenses, we tested the expression of GSH1 (glutamylcysteine synthetase), SOD1 (cooper/zinc superoxide dismutase), CTT1 (cytosolic catalase T), GPX2 (2-Cys peroxiredoxin), TRR1 (thioredoxin reductase) and TRX2 (thioredoxin 2) by quantitative real-time PCR analyses. In the 


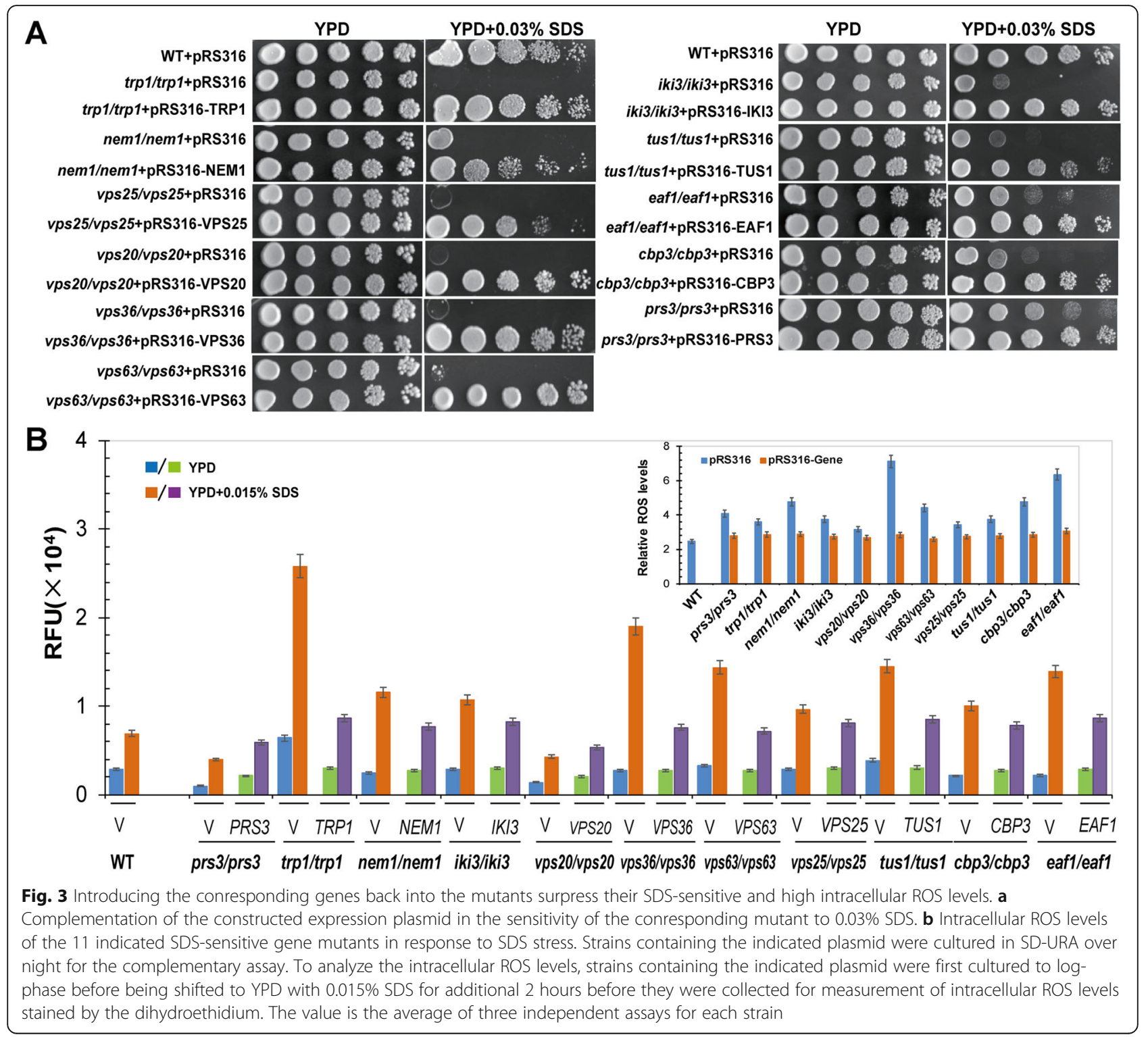

wild-type cells, the expression levels of GSH1, SOD1, CTT1 and GPX2 were significantly up-regulated after treatment with $0.015 \%$ SDS (Fig. 4), while no significant difference in the expression levels of TRR1 or TRX2 were observed when treated with or without SDS (Additional file 5: Fig. S4). Interestingly, both of the expression levels of SOD1 and CTT1 were reduced in the 11 mutants compared with wild type cells (Fig. 4b and c). In addition, the expression levels of GSH1 and GPX2 were also reduced in these mutants except the mutants for NEM1 and VPS25, or EAF1, respectively (Fig. 4a and d). To investigate the decreased expression of GSH1, SOD1, CTT1 and GPX2, we further analyzed the expression levels of these four genes in the wide type cells treated with 0.005 and $0.01 \%$ (Additional file 6: Fig. S5). We found that the expression levels of GSH1, SOD1, CTT1 and GPX2 were induced when the SDS concentrations were
0.01 and 0.015 , but remain unchanged or slightly induced when the SDS concentration was $0.005 \%$. It suggested that the expressions of the above four genes were dependent on the concentration of SDS. Overall, our results demonstrate that the decreased expression of GSH1, SOD1, CTT1 and GPX2 might be responsible for the high intracellular ROS levels accumulated in these mutants than wide type cells.

\section{Discussion}

SDS is considered as a generally recognized safe ingredient for food and hygiene products. However, safety concern arises as oral ulcer or skin irritation was reported to be caused by products containing SDS in recent studies [46, 47]. S. cerevisiae, a budding yeast used in brewing beer and baking, is a singlecelled eukaryote used extensively in laborary due to 


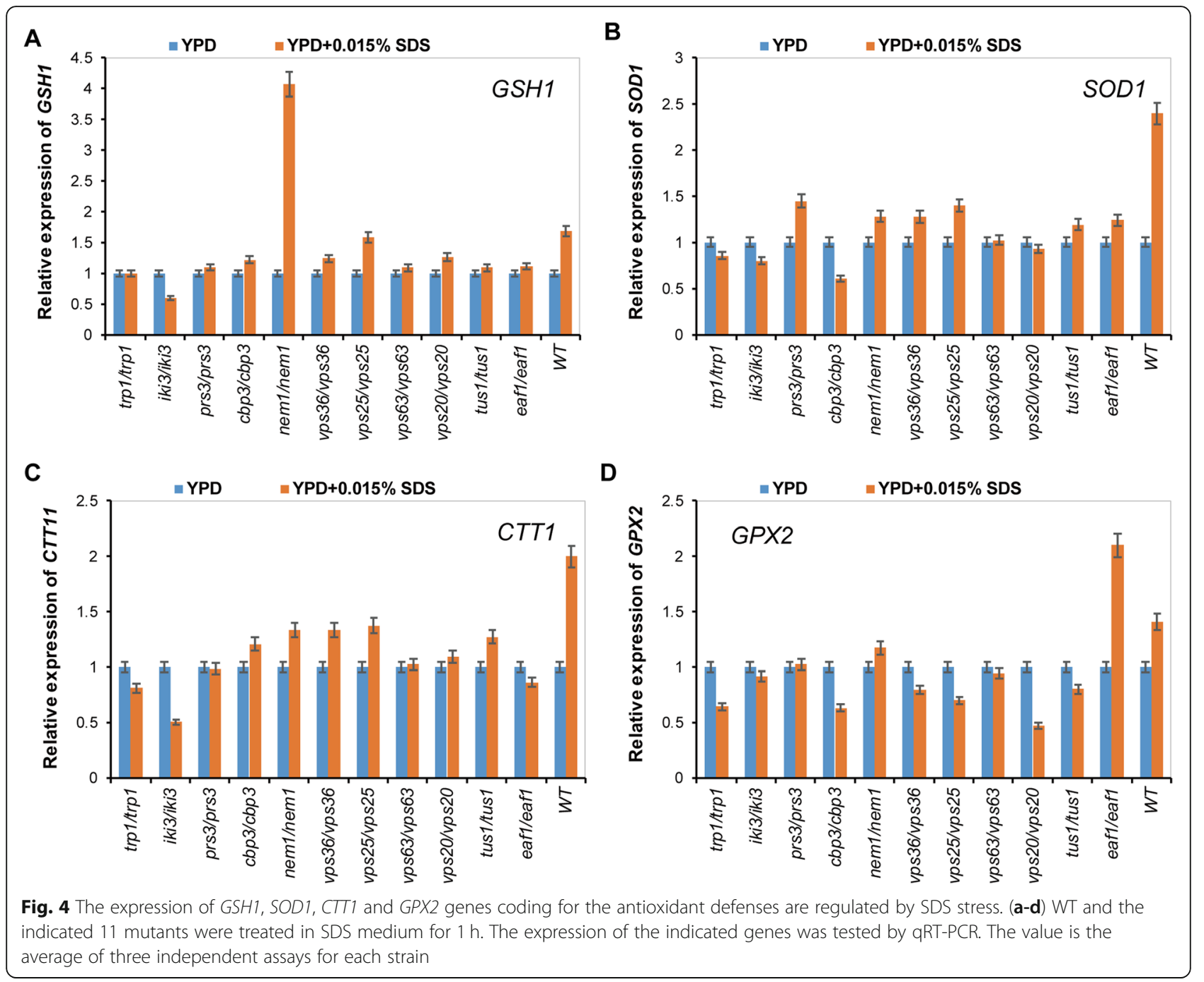

the fact that its genome has been sequenced and its genetics are easily manipulated. Here, we used S. cerevisiae to examiner the genome-wide SDS stress on eukaryotes and identified 108 SDS-sensitive mutants from the yeast nonessential gene deletion library, representing 2.3\% of the screened 4757 gene deletion mutants. Previous study reported that 295 ORFs were up-regulated and 118 ORFs were down-regulated aftert SDS treatment, and the functional classifications of these genes were involved in a number of major cellular processes, including metabolism, protein sorting, transcription, cellular transport and biogenesis, DNA and protein synthesis, cellular communication / signal transduction and ionic homeostasis, etc. [2]. Interestingly, The 108 SDS-sensitive genes encoded proteins that are also involved in many of these cellular processes.

A significant aspect of SDS toxicity may be related to its effect on biological membranes that SDS may penetrate the membrane through pores in the yeast cell wall and destroy the membrane [48]. For example, SDS is used as a perturbing agent to cell wall integrity, and through MPT5 and SSD1 signaling pathway SDS can result in sensitivity to changes in external osmolarity, defect budding, and cell lysis [49]. Our results support this by showing that mutants for six genes (TUS1, ROM2, SIT4, TOH1, SLG1 and LAS21) involved in the process of cell wall integrity were sensitive to SDS (Table 1 and Fig. 1). Interstingly, the intracellular ROS levels in these six mutants were all higher than wide type cells when cells were teated with SDS, indicating their crucial role in maintaining the cell wall integrity under SDS stress in S. cerevisiae.

Beside the stress on cell wall, SDS also introduced stresses on intracellular sorting and delivery of soluble vacuolar proteins. The largest functional category (28) of these identified SDS-sensitive genes are of cellular transport, transport facilities and transport routes. 
Vacuolar protein sorting (VPS) genes involved in vesicle transport to vacuoles play an important role in segregating molecules into distinct organelles and even affect the telomere length regulation [50]. Vacuolar $\mathrm{H}(+)$-ATPase (V-ATPase) localized in the vacuole membrane (V-ATPase) is composed of the catalytic V1 subcomplex and the proton-translocating membrane V0 subcomplex [51]. It has a crucial role in the vacuolar system and acidification of the vacuole and other internal compartments including the whole secretory pathway [52]. The V1 complex is composed of at least eight subunits (A-H) encoded by eight $V M A$ (Vacuolar Membrane ATPase) genes: VMA1, VMA2, VMA4, VMA5, VMA7, VMA8, VMA10 and $V M A 13$, respectively. The V0 complex is composed of at least five subunits (a, c, d, c' and c") encoded by six VMA genes: VPH1, STV1, VMA3, VMA6, VMA11, and VMA16, respectively. Three genes VMA12, $V M A 21$ and VMA22 encode proteins that are required for the biogenesis of a functional V-ATPase [51]. Mutants for the VMA genes showed growth defects in response to oxidative stress, such as $\mathrm{H}_{2} \mathrm{O}_{2}$ [53]. In present study, 15 SDS-sensitive genes involved in the VPS pathway and four SDS-sensitive genes involved in V-ATPase function have been identified sensitive to SDS stress. We speculate that the absence of these genes might reduce the supply of cell wall and/or cell membrane components, leading to cell membrane damage or defects in cell wall structure in the SDS stress.

The expression of about 65 genes involved in the carbon metabolism were induced by SDS stress, including genes related to amino acid metabolism, Ccompound and carbohydrate metabolism, lipid, fatty acid, and isoprenoid metabolism, vitamin, cofactor, and prosthetic group metabolism, and nucleotide metabolism [2, 54]. Mutants for a large group of 16 genes involved in metabolism is revealed to be sensitive to SDS stress in the present study, including six genes related to amino acids metabolism (ARO1, ARO2, ARO7, TRP1, TRP5, PRS3 and THR4), four genes related to lipid and fatty acid metabolism (ARG82, ELO3, IPK1, and ERG3), four genes involved in nucleotide metabolism (GRR1, REG1, ELM1 and $A F T 1$ ), and one gene associated with carbohydrate metabolism (NRK1) (Table 1 and Fig. 1). In a previous study, it has been showed that the cell membrane damage trigged by SDS stress was independent of Cell Wall Integrity signaling pathway, and the biosynthesis of tryptophan and tyrosine played an important role in the SDS-induced plasma membrane stress response [45]. It is might explain why the six mutants for ARO1, ARO2, ARO7, TRP1,, PRS3 and TRP5, involved in the synthesis of aromatic amino acids and tryptophan, were sensitive to SDS toxicity. In addition, lipid and fatty acid metabolism has significant role in maintaining the structures of cell membrane and cell wall, nucleotide metabolism is related to the processes of DNA synthesis, cell division and DNA repair, while carbohydrate metabolism is associated to cell growth and many other cellular activities. Moreover, it has been previously reported that, cell wall defects led to cells sensitive to SDS stress for a weakened cell wall allows it to penetrate more easily [8]. Therefore, it is not surprising that deletion mutants for the other ten genes involved in the above metabolism functions are sensitive to SDS stress.

Another concern with SDS toxicity has been its carcinogenicity; no evidence shows SDS-related tumorigenicity or carcinogenicity in early official review. SDS was extensively tested for genetic toxicity. Tests with SDS in bacterial or in mammalian systems (in vitro and in vivo) show no indication of genotoxicity with or without metabolic activation [55]. Published reports suggest that SDS has low acute mammalian toxicity and no known chronic effects. However, we have identified 14 SDS-sensitive genes of $S$. cerevisiae are involved in cell cycle and DNA processing in our study, though further investigation is required to clarify the significance.

Finally, we examined the intracellular ROS levels under SDS stress. Increased ROS level may result in significant damage to cell structures and constant high ROS level is known as oxidative stress. Most significantly, ROS is considered to damage DNA or RNA of cells. Under the SDS treatment, we observed most mutants (85/108) increased the intracellular ROS levels comparing with the wild-types, consistent with their being-affected growth. We pick up $11 \mathrm{mu}$ tants for PRS3, TRP1, NEM1, EAF1, IKI3, CBP3, VPS20, VPS36, VPS63, VPS25, and TUS1, which accumulated higher relative ROS levels than that of wildtype cells under SDS treatment (Fig. 2), to investigate the mechanism of oxidative damage induced by SDS stress. We have shown that the expression of some antioxidant defenses genes were down-regulated by SDS stress in these mutants. It suggests that some of the SDS-sensitive genes might be involved in maintaining the redox balance under SDS treatment. However, some mutants reduced its ROS production, the genes involved in these mutants may be related the detoxification of SDS by yeast cells. Another interesting result of our study is that six mutants for ARG82, TRP5, GRR1, MSH1, LAS21, and YNL296W, accumulated lower intracellular ROS levels under $0.015 \%$ SDS treatment when compared with no SDS treatment. They could also play a role in detoxification of SDS by yeast cells, but further investigations are needed. 


\section{Conclusions}

To study the SDS toxicity we have performed a genomewide screen for mutants that are sensitive to $0.03 \%$ SDS. It is demonstrated that the intracellular ROS levels in 85 of the identified 108 SDS-sensitive mutants were significantly higher than that of the wild-type BY4743 cells in response to SDS stress. In addition, the expression levels of genes involved in antioxidant defenses suggest that SDS might generate oxidative damage by regulating these genes, leading to cells sensitive to SDS stress. Taken together, our present study provides a potential way to understand the detoxification mechanisms of SDS by yeast cells.

\section{Methods}

\section{Strains, media and culture conditions}

All S. cerevisiae strains were derived from the S288C genetic background. The homozygous diploid deletion mutant library were purchased from Invitrogen Inc. [http://clones.invitrogen. $\mathrm{com} /]$ and was frozen at $-80{ }^{\circ} \mathrm{C}$ in 96 -well microtitre plates in $15 \%$ glycerol in liquid YPD medium (1\% yeast extract, $2 \%$ peptone, $2 \%$ glucose). SD-URA media $(0.17 \%(\mathrm{w} / \mathrm{v})$ yeast nitrogen base, $2 \%(\mathrm{w} / \mathrm{v})$ glucose, $0.5 \%$ ammonia sulfate, adding $1 / 10 \mathrm{~mL}$ amino acid mixture without uracil) was used to culture yeast cells for plasmid selection. Solid media were produced by adding $2 \%(\mathrm{w} / \mathrm{v})$ agar when necessary. SDS was purchased from Sangon Biotech (Shanghai, China), and Dihydroethidium was purchased from Sigma (Beijing, China).

\section{Primary screen for mutants involved in SDS toxicity}

Prior to the primary screen for SDS-sensitive mutations, the deletion mutant library was first transferred to fresh liquid YPD medium and cultured at $30^{\circ} \mathrm{C}$. Then each mutant strains were transferred to fresh liquid YPD medium with or without $0.015 \%$ SDS, respectively, and cultured at $30{ }^{\circ} \mathrm{C}$ for about 6 to $12 \mathrm{~h}$. The growth rates of each mutant in YPD medium with and without $0.015 \%$ SDS were measured at $\mathrm{OD}_{600}$ to determine the SDS-sensitivity in the primary screen. The SDS sensitive strains showed reduced growth and was defined as mutants with a relative $\mathrm{OD}_{600}$ reduced by more than $30 \%$ in liquid YPD medium containing supplemented SDS but not in liquid YPD medium without supplemented SDS as compared to that of the wild-type.

\section{Phenotypic analysis by spot dilution growth assays}

The identified mutants that appeared sensitive were subjected to the secondary screen retested by spot dilution growth assays. In brief, each mutants were cultured overnight in liquid YPD at $30^{\circ} \mathrm{C}$ and then were spotted onto YPD plates with or without $0.03 \%$ SDS in comparison to the wild type BY4743 strain.

To further confirm the SDS sensitivity of mutants for PRS3, TRP1, NEM1, EAF1, IKI3, CBP3, VPS20, VPS36, VPS63, VPS25, and TUS1 genes, we introduced the pRS316 vector and pRS316 vector expressing the conresponding genes back into mutants for PRS3, TRP1, NEM1, EAF1, IKI3, CBP3, VPS20, VPS36, VPS63, VPS25, and TUS1. The sensitivity to SDS of the transformants was examined on YPD and YPD $+0.03 \%$ SDS plates using the above serial dilution assay method.

\section{DNA manipulations}

To express the TRP1 gene in the plasmid pRS316, the DNA fragment which contains the promoter, ORF and terminator region, was first amplified with primers TRP1-F and TRP1-R (Additional file 1: Table S1), and were cloned into the BamHI and HindIII sites of pRS316 to yield pRS316-TRP1. The other plasmids of pRS316- IKI3, pRS316-PRS3, pRS316CBP3, pRS316-NEM1, pRS316-VPS36, pRS316-VPS25, pRS316-VPS63, pRS316-VPS20, pRS316-TUS1 and pRS316$E A F 1$ were all constructed by the same method described above. All the inserts were confirmed by DNA sequencing.

\section{Oxidative stress assay for SDS-sensitive mutants}

To determine the cellular oxidative stress of the SDSsensitive mutants, we tested the intracellular ROS level by the dihydroethidium as previously described [56]. Briefly, overnight cell cultures were inoculated in YPD to an optical density $\mathrm{OD}_{600}=0.1$, grown to middle log phase, and split into two aliquots with or without $0.015 \%$ SDS and were grown for $2 \mathrm{~h}$. Then about $5 \times 10^{6}$ cells were harvested by centrifugation and resuspended in $250 \mu \mathrm{l}$ PBS with $2.5 \mu \mathrm{g} / \mathrm{ml} \mathrm{DHE}$, and incubated in the dark for 30 min. The relative fluorescence units (RFU) were tested by a fluorescence reader (Synergy ${ }^{\text {TM }}$ H4, BioTek).

\section{RNA extraction and quantitative PCR analysis}

The mutants were first grown to middle log phase $\left(\mathrm{OD}_{600}=0.6-1.0\right)$, and then they were grown in the presence or absence of $0.015 \%$ SDS for $1 \mathrm{~h}$. The total RNA was extracted by hot phenol method. The genomic DNA was first removed from the total RNA with RNase-free DNase I. The first-strand cDNA synthesis was performed using the Primer Script RT reagent kit (Cwbiotech, China) according to the manufacturer's instructions. The expression mRNA levels of TRR1, TRX2, GSH1, SOD1, CTT1 and GPX2 were detected by quantitative PCR (qPCR) as described previously [57] (Additional file 1: Table S1). Each reaction was carried out in triplicate.

\section{Enrichment analysis for the identified genes}

The web-based tool (http://metascape.org/gp/index. $\mathrm{html} / \mathrm{main} / \mathrm{step} 1)$ was used for enrichment analysis of SDS-sensitive genes. $p$-value $<0.01$, min overlap genes $=$ 3 , and min enrichment factor $>1.5$ were set as the cutoff criteria and the significance was ranked by enrichment score $(-\log 10$ (P-value)). 


\section{Supplementary information}

Supplementary information accompanies this paper at https://doi.org/10. 1186/s12866-020-1721-2

\section{Additional file 1 : Table S1. Primers used in this study.}

Additional file 2 : Fig. S1. Genotype confirmation of the 108 gene deletion mutants by PCR. Cells of the 108 gene mutants were grown overnight in YPD medium at $30^{\circ} \mathrm{C}$ and then collected for DNA extraction. PCR was performed with genomic DNA of each of these mutants with the primer located at the upstream of its open reading frame and the reverse primer KanMX4-R from the internal sequence of the KanMX4. PCR products were separated on $1 \%$ agarose gel, and sizes of the DNA marker were indicated on the left or right of the gel.

Additional file 3 : Fig. S2. Meta-enrichment analysis summary of SDSsensitive genes. Heatmap of the top 16 enriched $\mathrm{GO}$ terms. For GO terms, each band represents one enriched term coloured according to its -log $10 p$-value. The dominant term within each group is used as a group heading.

Additional file 4 : Fig. S3. Intracellular ROS levels of 108 SDS-sensitive gene mutants in response to SDS stress. a: Metabolism; b: Cell cycle and DNA Processing; c: Transcription; d: Protein with Binding Function or Cofactor Requirement (structural or catalytic); e: Cellular Transport, Transport Facilities and Transport Routes; f: Biogenesis of cellular components; g: Cell wall integrity and osmotic stress response; $h$ : Unclassified Proteins. Log-phase cells were grown with or without $0.015 \%$ SDS for two hours before they were collected for measurement of intracellular ROS levels stained by the dihydroethidium. The intracellular ROS levels of these SDSsensitive mutants were listed according to their categories in comparison to that of wild type cell BY4743. The value is the average of three independent assays for each strain.

Additional file $\mathbf{5}$ : Fig. S4. The expression of TRR1 and TRX2 under SDS stress. (A-B) WT and the indicated 11 mutants were treated to SDS medium for $1 \mathrm{~h}$. The expression of the indicated genes was tested by qRT-PCR. The value is the average of three independent assays for each strain.

Additional file 6 : Fig. S5. The expression levels of GSH1, SOD1, CTT1 and GPX2 genes in response to different concentrations of SDS in the wide type BY4743 cells. The expression of the indicated genes was tested by qRT-PCR. The value is the average of three independent assays for each strain.

\section{Abbreviations}

CWI: Cell wall integrity; QPCR: Quantitative PCR; RFU: Relative fluorescence units; ROS: Reavtive oxygen species; SDS: Sodium dodecyl sulfate; VMA: Vacuolar membrane ATPase; VPS: Vacuolar protein sorting

\section{Acknowledgements}

We thank the Test Platform for Large Instruments and Equipment of School of Biotechnology and the State Key Laboratory of Food Science and Technology for the technical supports.

\section{Authors' contributions}

ZYY designed the experiment and revised the manuscript. CCL and CZF performed the experiment. CCL and YPB wrote and revised the manuscript. We confirm that the final version manuscript has been read and approved by all named authors.

\section{Funding}

This work was supported by the Natural Science Foundation of Jiangsu Province (BK20181345) and the Open Foundation of Jiangsu Key Laboratory of Industrial Biotechnology (KLIB-KF201807). None of the funders had any role in designing and/or conducting of the study; collection, management, analysis and interpretation of the data; and preparation, review or approval of the manuscript.

\section{Availability of data and materials}

All data generated or analysed during this study are included in this published article and its supplementary information files.
Ethics approval and consent to participate

Not applicable.

\section{Consent for publication}

Not applicable.

\section{Competing interests}

The authors declare that they have no competing interests.

\section{Author details}

${ }^{1}$ National Engineering Laboratory for Cereal Fermentation Technology (NELCF), Jiangnan University, 1800 Lihu Road, Wuxi 214122, Jiangsu, China. ${ }^{2}$ Jiangsu Provincial Research Center for Bioactive Product Processing Technology, Jiangnan University, 1800 Lihu Road, Wuxi 214122, Jiangsu, China. ${ }^{3}$ College of Animal Science and Technology, Yangzhou University, Yangzhou 225009, Jiangsu, China.

Received: 21 August 2019 Accepted: 6 February 2020

Published online: 17 February 2020

\section{References}

1. Wang Y, Zhang Y, Li X, Sun M, Wei Z, Wang Y, Gao A, Chen D, Zhao X, Feng $X$. Exploring the effects of different types of surfactants on zebrafish embryos and larvae. Sci Rep. 2015:5:10107.

2. Sirisattha S, Momose Y, Kitagawa E, Iwahashi H. Toxicity of anionic detergents determined by Saccharomyces cerevisiae microarray analysis. Water Res. 2004;38(1):61-70.

3. Bondi CA, Marks JL, Wroblewski LB, Raatikainen HS, Lenox SR, Gebhardt KE. Human and environmental toxicity of sodium lauryl sulfate (SLS): evidence for safe use in household cleaning products. Environ Health Insights. 2015;9: 27-32.

4. Messina CM, Faggio C, Laudicella VA, Sanfilippo M, Trischitta F, Santulli A. Effect of sodium dodecyl sulfate (SDS) on stress response in the Mediterranean mussel (Mytilus Galloprovincialis): regulatory volume decrease (Rvd) and modulation of biochemical markers related to oxidative stress. Aquat Toxicol. 2014;157:94-100.

5. Bindu PC, Babu P. Surfactant-induced lipid peroxidation in a tropical euryhaline teleost Oreochromis mossambicus (tilapia) adapted to fresh water. Indian J Exp Biol. 2001;39(11):1118-22.

6. Igual JC, Johnson $\mathrm{AL}$, Johnston $\mathrm{LH}$. Coordinated regulation of gene expression by the cell cycle transcription factor Swi4 and the protein kinase C MAP kinase pathway for yeast cell integrity. EMBO J. 1996;15(18):5001-13.

7. Levin DE. Regulation of cell wall biogenesis in Saccharomyces cerevisiae: the cell wall integrity signaling pathway. Genetics. 2011;189(4):1145-75.

8. Levin DE. Cell wall integrity signaling in Saccharomyces cerevisiae. Microbiol Mol Biol Rev. 2005:69(2):262-91.

9. Morimoto $Y$, Tani M. Synthesis of mannosylinositol phosphorylceramides is involved in maintenance of cell integrity of yeast Saccharomyces cerevisiae. Mol Microbiol. 2015;95(4):706-22.

10. Gubbens J, Slijper M, de Kruijff B, de Kroon AIPM. Protein complexes in bacterial and yeast mitochondrial membranes differ in their sensitivity towards dissociation by SDS. Biochim Biophys Acta. 2008;1784(12):2012-8.

11. Madeo F, Frohlich E, Ligr M, Grey M, Sigrist SJ, Wolf DH, Frohlich KU. Oxygen stress: a regulator of apoptosis in yeast. J Cell Biol. 1999;145(4):75767

12. Fleury C, Mignotte B, Vayssiere $J$. Mitochondrial reactive oxygen species in cell death signaling. Biochimie. 2002;84(2-3):131-41.

13. Gao Q, Liou LC, Ren Q, Bao X, Zhang Z. Salt stress causes cell wall damage in yeast cells lacking mitochondrial DNA. Microb Cell. 2014;1(3):94-9.

14. Luo $\mathrm{C}$, Cao $\mathrm{CL}$, Jiang $\mathrm{LH}$. The endosomal sorting complex required for transport (ESCRT) is required for the sensitivity of yeast cells to nickel ions in Saccharomyces cerevisiae. FEMS Yeast Res. 2016;16(3):1-8.

15. Bowers K, Stevens TH. Protein transport from the late Golgi to the vacuole in the yeast Saccharomyces cerevisiae. Biochim Biophys Acta. 2005;1744(3): 438-54.

16. Kane PM. The where, when, and how of organelle acidification by the yeast vacuolar H+-ATPase. Microbiol Mol Biol Rev. 2006;70(1):177-91.

17. Nishi T, Forgac M. The vacuolar $(\mathrm{H}+)$-ATPases--nature's most versatile proton pumps. Nat Rev Mol Cell Biol. 2002;3(2):94-103.

18. Ho MN, Hill KJ, Lindorfer MA, Stevens TH. Isolation of vacuolar membrane $\mathrm{H}(+)$-ATPase-deficient yeast mutants; the VMA5 and VMA4 genes are 
essential for assembly and activity of the vacuolar H(+)-ATPase. J Biol Chem. 1993:268(1):221-7.

19. Keenan Curtis K, Kane PM. Novel vacuolar H+-ATPase complexes resulting from overproduction of Vma5p and Vma13p. J Biol Chem. 2002;277(4): 2716-24.

20. Szczypka MS, Zhu Z, Silar P, Thiele DJ. Saccharomyces cerevisiae mutants altered in vacuole function are defective in copper detoxification and ironresponsive gene transcription. Yeast. 1997;13(15):1423-35.

21. Graham LA, Stevens TH. Assembly of the yeast vacuolar protontranslocating ATPase. J Bioenerg Biomembr. 1999;31(1):39-47.

22. Schweiggert J, Stevermann L, Panigada D, Kammerer D, Liakopoulos D. Regulation of a spindle positioning factor at kinetochores by SUMOtargeted ubiquitin ligases. Dev Cell. 2016;36(4):415-27.

23. van de Pasch LA, Miles AJ, Nijenhuis W, Brabers NA, van Leenen D, Lijnzaad P, Brown MK, Ouellet J, Barral Y, Kops GJ, et al. Centromere binding and a conserved role in chromosome stability for SUMO-dependent ubiquitin ligases. PLoS One. 2013;8(6):e65628.

24. Schweiggert J, Stevermann L, Panigada D, Liakopoulos D. Regulation of a spindle positioning factor at kinetochores by SUMO-targeted ubiquitin ligases. Dev cell. 2016;36(4):415-27.

25. Mookerjee SA, Lyon HD, Sia EA. Analysis of the functional domains of the mismatch repair homologue Msh1p and its role in mitochondrial genome maintenance. Curr Genet. 2005;47(2):84-99.

26. Page N, Gerard-Vincent M, Menard P, Beaulieu M, Azuma M, Dijkgraaf GJ, Li H, Marcoux J, Nguyen T, Dowse T, et al. A Saccharomyces cerevisiae genome-wide mutant screen for altered sensitivity to K1 killer toxin. Genetics. 2003;163(3):875-94.

27. Trujillo KM, Roh DH, Chen L, Van Komen S, Tomkinson A, Sung P. Yeast xrs2 binds DNA and helps target rad50 and mre11 to DNA ends. J Biol Chem. 2003;278(49):48957-64.

28. Bungard D, Reed M, Winter E. RSC1 and RSC2 are required for expression of mid-late sporulation-specific genes in Saccharomyces cerevisiae. Eukaryot Cell. 2004;3(4):910-8.

29. Auger A, Galarneau L, Altaf M, Nourani A, Doyon Y, Utley RT, Cronier D, Allard S, Cote J. Eaf1 is the platform for NuA4 molecular assembly that evolutionarily links chromatin acetylation to ATP-dependent exchange of histone H2A variants. Mol Cell Biol. 2008;28(7):2257-70.

30. Jonsson ZO, Jha S, Wohlschlegel JA, Dutta A. Rvb1p/Rvb2p recruit Arp5p and assemble a functional Ino80 chromatin remodeling complex. Mol Cell. 2004;16(3):465-77.

31. Mayer ML, Gygi SP, Aebersold R, Hieter P. Identification of RFC (Ctf18p, (tf8p, Dcc 1p): an alternative RFC complex required for sister chromatid cohesion in S. cerevisiae. Mol Cell. 2001;7(5):959-70.

32. Hanna JS, Kroll ES, Lundblad V, Spencer FA. Saccharomyces cerevisiae CTF18 and CTF4 are required for sister chromatid cohesion. Mol Cell Biol. 2001; 21(9):3144-58.

33. Bleackley MR, Young BP, Loewen CJ, MacGillivray RT. High density array screening to identify the genetic requirements for transition metal tolerance in Saccharomyces cerevisiae. Metallomics. 2011;3(2):195-205.

34. Huang D, Friesen $H$, Andrews B. Pho85, a multifunctional cyclin-dependent protein kinase in budding yeast. Mol Microbiol. 2007;66(2):303-14.

35. Outten CE, Falk RL, Culotta VC. Cellular factors required for protection from hyperoxia toxicity in Saccharomyces cerevisiae. Biochem J. 2005;388(Pt 1): 93-101.

36. Cai H, Kauffman S, Naider F, Becker JM. Genomewide screen reveals a wide regulatory network for di/tripeptide utilization in Saccharomyces cerevisiae. Genetics. 2006;172(3):1459-76.

37. Dudley AM, Janse DM, Tanay A, Shamir R, Church GM. A global view of pleiotropy and phenotypically derived gene function in yeast. Mol Syst Biol. 2005;1:2005 0001.

38. Kim Y, Gentry MS, Harris TE, Wiley SE, Lawrence JC Jr, Dixon JE. A conserved phosphatase cascade that regulates nuclear membrane biogenesis. Proc Natl Acad Sci U S A. 2007;104(16):6596-601.

39. Lenoir G, Williamson P, Puts CF, Holthuis JC. Cdc50p plays a vital role in the ATPase reaction cycle of the putative aminophospholipid transporter Drs $2 p$. J Biol Chem. 2009;284(27):17956-67.

40. Henstrand JM, Schaller A, Braun M, Amrhein N, Schmid J. Saccharomyces cerevisiae chorismate synthase has a flavin reductase activity. Mol Microbiol. 1996;22(5):859-66.

41. Tkach JM, Yimit A, Lee AY, Riffle M, Costanzo M, Jaschob D, Hendry JA, Ou J, Moffat J, Boone C, et al. Dissecting DNA damage response pathways by analysing protein localization and abundance changes during DNA replication stress. Nat Cell Biol. 2012;14(9):966-76.

42. Ball SG, Wickner RB, Cottarel G, Schaus M, Tirtiaux C. Molecular cloning and characterization of ARO7-OSM2, a single yeast gene necessary for chorismate mutase activity and growth in hypertonic medium. Mol Gen Genet. 1986;205(2):326-30.

43. Leng G, Song K. Watch out for your TRP1 marker: the effect of TRP1 gene on the growth at high and low temperatures in budding yeast. FEMS Microbiol Lett. 2016;363(10):fnw093.

44. Zalkin $\mathrm{H}$, Yanofsky C. Yeast gene TRP5: structure, function, regulation. J Biol Chem. 1982;257(3):1491-500.

45. Schroeder L, Ikui AE. Tryptophan confers resistance to SDS-associated cell membrane stress in Saccharomyces cerevisiae. PLoS One. 2019;14(3): e0199484.

46. Lippert F. An introduction to toothpaste - its purpose, history and ingredients. Monogr Oral Sci. 2013;23:1-14.

47. Marrakchi S, Maibach HI. Sodium lauryl sulfate-induced irritation in the human face: regional and age-related differences. Skin Pharmacol Physiol. 2006:19(3):177-80.

48. Levin DE, Bartlett-Heubusch. E. Mutants in the S. cerevisiae PKC1 gene display a cell cycle-specific osmotic stability defect. J Cell Biol. 1992;116(5): 1221-9.

49. Kaeberlein M, Guarente L. Saccharomyces cerevisiae MPT5 and SSD1 function in parallel pathways to promote cell wall integrity. Genetics. 2002;160(1):8395.

50. Rog O, Smolikov S, Krauskopf A, Kupiec M. The yeast VPS genes affect telomere length regulation. Curr Genet. 2005;47(1):18-28.

51. Graham LA, Powell B, Stevens TH. Composition and assembly of the yeast vacuolar H(+)-ATPase complex. J Exp Biol. 2000;203(Pt 1):61-70.

52. Corbacho I, Teixido F, Olivero I, Hernandez LM. Dependence of Saccharomyces cerevisiae Golgi functions on V-ATPase activity. FEMS Yeast Res. 2012;12(3):341-50.

53. Milgrom E, Diab H, Middleton F, Kane PM. Loss of vacuolar protontranslocating ATPase activity in yeast results in chronic oxidative stress. J Biol Chem. 2007;282(10):7125-36.

54. Nickerson KW, Aspedon A. Detergent-shock response in enteric bacteria. Mol Microbiol. 1992;6(8):957-61.

55. Cohen A, Nelson $\mathrm{H}$, Nelson $\mathrm{N}$. The family of SMF metal ion transporters in yeast cells. J Biol Chem. 2000;275(43):33388-94.

56. Buttner S, Eisenberg T, Carmona-Gutierrez D, Ruli D, Knauer H, Ruckenstuhl C, Sigrist C, Wissing S, Kollroser M, Frohlich KU, et al. Endonuclease G regulates budding yeast life and death. Mol Cell. 2007;25(2):233-46.

57. Zhao YY, Cao CL, Liu YL, Wang J, Li J, Li SY, Deng Y. Identification of the genetic requirements for zinc tolerance and toxicity in Saccharomyces cerevisiae. G3 (Bethesda). 2019. https://doi.org/10.1534/g3.119.400933.

\section{Publisher's Note}

Springer Nature remains neutral with regard to jurisdictional claims in published maps and institutional affiliations.

Ready to submit your research? Choose BMC and benefit from

- fast, convenient online submission

- thorough peer review by experienced researchers in your field

- rapid publication on acceptance

- support for research data, including large and complex data types

- gold Open Access which fosters wider collaboration and increased citations

- maximum visibility for your research: over $100 \mathrm{M}$ website views per year

At $\mathrm{BMC}$, research is always in progress.

Learn more biomedcentral.com/submissions 